manuscript No.

(will be inserted by the editor)

\title{
Some cubics with finite-dimensional motive
}

\author{
Robert Laterveer
}

Received: date / Accepted: date

\begin{abstract}
This small note presents in any dimension a family of cubics that have finite-dimensional motive (in the sense of Kimura). As an illustration, we verify a conjecture of Voevodsky for these cubics, and a conjecture of Murre for the Fano variety of lines of these cubics.
\end{abstract}

Keywords Algebraic cycles · Chow groups · motives $\cdot$ finite-dimensional motives $\cdot$ cubics

Mathematics Subject Classification (2010) $14 \mathrm{C} 15,14 \mathrm{C} 25,14 \mathrm{C} 30$.

\section{Introduction}

The notion of finite-dimensional motive, developed independently by Kimura and O'Sullivan [15], [1], [21], [12], [8] has given important new impetus to the study of algebraic cycles. To give but one example: thanks to this notion, we now know the Bloch conjecture is true for surfaces of geometric genus zero that are rationally dominated by a product of curves [15]. It thus seems worthwhile to find concrete examples of varieties that have finite-dimensional motive, this being (at present) one of the sole means of arriving at a satisfactory understanding of Chow groups.

The present note aims to contribute something to the list of examples of varieties with finite-dimensional motive, by considering cubic hypersurfaces. In any dimension, there is one famous cubic known to have finite-dimensional motive: the Fermat cubic

$$
\left(x_{0}\right)^{3}+\left(x_{1}\right)^{3}+\cdots+\left(x_{n+1}\right)^{3}=0 .
$$

The Fermat cubic has finite-dimensional motive because it is rationally dominated by a product of curves, and the indeterminacy locus is again of Fermat type [28]. In [17], I proved finite-dimensionality for a certain 10-dimensional family of cubic fourfolds. The main result of this note gives, for any dimension, a family of cubics (containing the Fermat cubic) with finite-dimensional motive:

Theorem (=theorem 5 Let $X \subset \mathbb{P}^{n+1}(\mathbb{C})$ be a smooth cubic defined by an equation

$$
f_{0}\left(x_{0}, \ldots, x_{4}\right)+f_{1}\left(x_{5}, \ldots, x_{9}\right)+\cdots+f_{r}\left(x_{5 r}, \ldots, x_{5 r+4}\right)+f_{r+1}\left(x_{5(r+1)}, \ldots, x_{n+1}\right)=0,
$$

where $f_{0}, \ldots, f_{r}$ define smooth cubics of dimension 3 , and $f_{r+1}$ defines a smooth cubic of dimension $<3$ (i.e., $r=\left\lfloor\frac{n+1}{5}\right\rfloor$ ). Then $X$ has finite-dimensional motive.

CNRS - IRMA, Université de Strasbourg

7 rue René Descartes

67084 Strasbourg cedex

France

E-mail: laterv@math.unistra.fr 
The proof is an elementary argument: employing the inductive structure exhibited by Shioda [28], [14], one reduces theorem 5 to the main result of [17].

To illustrate how nicely the concept of finite-dimensionality allows to understand algebraic cycles, we provide an application to a conjecture of Voevodsky concerning smash-equivalence [33]:

Corollary (=corollary 14) Let $X$ be a cubic as in theorem 5 and suppose $n=\operatorname{dim} X$ is odd. Then numerical equivalence and smash-equivalence coincide for all algebraic cycles on $X$.

(For the definition of smash-equivalence, cf. definition[11)

We also establish the existence of a Chow-Künneth decomposition (as conjectured by Murre [20]) for the Fano variety of lines on a cubic as in theorem 5 , this is corollary 9

Conventions All varieties will be projective irreducible varieties over $\mathbb{C}$.

For smooth $X$ of dimension $n$, we will denote by $A^{j}(X)=A_{n-j}(X)$ the Chow group $C H^{j}(X) \otimes \mathbb{Q}$ of codimension $j$ cycles under rational equivalence. The notation $A_{\text {num }}^{j}(X)$ and $A_{\otimes}^{j}(X)$ will denote the subgroup of numerically trivial resp. smash-nilpotent cycles. The category $\mathcal{M}_{\text {rat }}$ will denote the (contravariant) category of Chow motives [24], [21] over $\mathbb{C}$. For a smooth projective variety, $h(X)=\left(X, \Delta_{X}, 0\right)$ will denote its motive in $\mathcal{M}_{\text {rat }}$.

\section{Finite-dimensionality}

We refer to [15], [1], [21], [8], [12] for the definition of finite-dimensional motive. An essential property of varieties with finite-dimensional motive is embodied by the nilpotence theorem:

Theorem 1 (Kimura [15]) Let $X$ be a smooth projective variety of dimension $n$ with finite-dimensional motive. Let $\Gamma \in A^{n}(X \times X)$ be a correspondence which is numerically trivial. Then there is $N \in \mathbb{N}$ such that

$$
\Gamma^{\circ N}=0 \quad \in A^{n}(X \times X)
$$

Actually, the nilpotence property (for all powers of $X$ ) could serve as an alternative definition of finitedimensional motive, as shown by a result of Jannsen [12, Corollary 3.9].

Conjecture 2 (Kimura [15]) All smooth projective varieties have finite-dimensional motive.

We are still far from knowing this, but at least there are quite a few non-trivial examples:

Remark 3 The following varieties have finite-dimensional motive: abelian varieties, varieties dominated by products of curves [15], K3 surfaces with Picard number 19 or 20 [22], surfaces not of general type with $p_{g}=0$ [7, Theorem 2.11], certain surfaces of general type with $p_{g}=0$ [7], [23], [34], Hilbert schemes of surfaces known to have finite-dimensional motive [4], generalized Kummer varieties [36, Remark 2.9(ii)], threefolds with nef tangent bundle [9] (an alternative proof is given in [30, Example 3.16]), fourfolds with nef tangent bundle [10], log-homogeneous varieties in the sense of [3] (this follows from [10, Theorem 4.4]), certain threefolds of general type [32, Section 8], varieties of dimension $\leq 3$ rationally dominated by products of curves [30, Example 3.15], varieties $X$ with $A_{A J}^{i}(X)=0$ for all $i$ [29. Theorem 4], products of varieties with finite-dimensional motive [15].

Remark 4 It is an embarrassing fact that up till now, all examples of finite-dimensional motives happen to lie in the tensor subcategory generated by Chow motives of curves, i.e. they are "motives of abelian type" in the sense of [30]. On the other hand, there exist many motives that lie outside this subcategory, e.g. the motive of a very general quintic hypersurface in $\mathbb{P}^{3}$ [6, 7.6]. 


\section{Main result}

Theorem 5 The following cubics have finite-dimensional motive (of abelian type): (i) a smooth cubic $X \subset \mathbb{P}^{5 r+4}(\mathbb{C})$ given by an equation

$$
f_{0}\left(x_{0}, \ldots, x_{4}\right)+f_{1}\left(x_{5}, \ldots, x_{9}\right)+\cdots+f_{r}\left(x_{5 r}, \ldots, x_{5 r+4}\right)=0,
$$

where the $f_{i}$ define smooth cubics;

(ii) a smooth cubic $X \subset \mathbb{P}^{5 r+5}(\mathbb{C})$ given by an equation

$$
f_{0}\left(x_{0}, \ldots, x_{4}\right)+f_{1}\left(x_{5}, \ldots, x_{9}\right)+\cdots+f_{r}\left(x_{5 r}, \ldots, x_{5 r+4}\right)+\left(x_{5 r+5}\right)^{3}=0,
$$

where the $f_{i}$ define smooth cubics;

(iii) a smooth cubic $X \subset \mathbb{P}^{5 r+6}(\mathbb{C})$ given by an equation

$$
f_{0}\left(x_{0}, \ldots, x_{4}\right)+f_{1}\left(x_{5}, \ldots, x_{9}\right)+\cdots+f_{r}\left(x_{5 r}, \ldots, x_{5 r+4}\right)+f_{r+1}\left(x_{5 r+5}, x_{5 r+6}\right)=0,
$$

where the $f_{i}$ define smooth cubics;

(iv) a smooth cubic $X \subset \mathbb{P}^{5 r+2}(\mathbb{C})$ given by an equation

$$
f_{0}\left(x_{0}, \ldots, x_{4}\right)+f_{1}\left(x_{5}, \ldots, x_{9}\right)+\cdots+f_{r-1}\left(x_{5 r-5}, \ldots, x_{5 r-1}\right)+f_{r}\left(x_{5 r}, x_{5 r+1}, x_{5 r+2}\right)=0,
$$

where the $f_{i}$ define smooth cubics;

(v) a smooth cubic $X \subset \mathbb{P}^{5 r+3}(\mathbb{C})$ given by an equation

$$
f_{0}\left(x_{0}, \ldots, x_{4}\right)+f_{1}\left(x_{5}, \ldots, x_{9}\right)+\cdots+f_{r-1}\left(x_{5 r-5}, \ldots, x_{5 r-1}\right)+f_{r}\left(x_{5 r}, \ldots, x_{5 r+3}\right)=0,
$$

where the $f_{i}$ define smooth cubics;

Proof The proof uses Shioda's inductive structure, in the guise of the following proposition (this is [14, Remark 1.10]):

Proposition 6 (Katsura-Shioda [14]) Let $Z \subset \mathbb{P}^{m_{1}+m_{2}}$ be a smooth hypersurface of degree d defined by an equation

$$
g_{1}\left(x_{0}, \ldots, x_{m_{1}}\right)+g_{2}\left(x_{m_{1}+1}, \ldots, x_{m_{1}+m_{2}}\right)=0 .
$$

Let $Z_{1}$ resp. $Z_{2}$ be the smooth hypersurfaces of dimension $m_{1}$ resp. $m_{2}-1$, defined as

$$
g_{1}\left(x_{0}, \ldots, x_{m_{1}}\right)+y^{d}=0,
$$

resp.

$$
g_{2}\left(x_{m_{1}+1}, \ldots, x_{m_{1}+m_{2}}\right)+z^{d}=0 .
$$

Then there exists a dominant rational map

$$
\phi: Z_{1} \times Z_{2} \rightarrow Z,
$$

and the indeterminacy of $\phi$ is resolved by blowing up the locus

$$
\left(Z_{1} \cap(y=0)\right) \times\left(Z_{2} \cap(z=0)\right) \subset Z_{1} \times Z_{2} .
$$

Let us first check theorem 5 is true for $r=0$. In case (i), this is clear since any cubic threefold has $A_{A J}^{*}(X)=0$ and so has finite-dimensional motive. In case (ii), the fourfold $X$ has finite-dimensional motive thanks to [17]. In case (iii), we use that any cubic fivefold has $A_{A J}^{*}(X)=0$ and so has finitedimensional motive. In cases (iv) and (v), the finite-dimensionality is again clear, since any curve and any del Pezzo surface has finite-dimensional motive.

Next, let us suppose theorem 5 is true for $r-1$, and let us prove this implies theorem 5 for $r$.

Let $X \subset \mathbb{P}^{5 r+4}$ be a cubic as in (i). According to proposition 6, there is a dominant rational map

$$
\phi: X_{1} \times X_{2} \rightarrow X,
$$


where

$$
\begin{aligned}
& X_{1}=\left\{f_{0}+f_{1}+\cdots+f_{r-1}+y^{3}=0\right\}, \\
& X_{2}=\left\{f_{r}+z^{3}=0\right\} .
\end{aligned}
$$

By induction, $X_{1}$ and $X_{2}$ have finite-dimensional motive. The indeterminacy of the rational map $\phi$ is resolved by blowing up $Y_{1} \times Y_{2} \subset X_{1} \times X_{2}$, where $Y_{1}=X_{1} \cap(y=0)$ and $Y_{2}=X_{2} \cap(z=0)$. By induction, $Y_{1}$ and $Y_{2}$ have finite-dimensional motive, and so $X$ (being dominated by something with finite-dimensional motive) has finite-dimensional motive.

Let $X \subset \mathbb{P}^{5 r+5}$ be a cubic as in (ii). According to proposition 6 , there is a dominant rational map

$$
\phi: \quad X_{1} \times X_{2} \rightarrow X,
$$

where

$$
\begin{aligned}
& X_{1}=\left\{f_{0}+f_{1}+\cdots+f_{r-1}+y^{3}=0\right\}, \\
& X_{2}=\left\{f_{r}+\left(x_{5 r+5}\right)^{3}+z^{3}=0\right\} .
\end{aligned}
$$

By induction, $X_{1}$ and $X_{2}$ have finite-dimensional motive. The indeterminacy of the rational map $\phi$ is resolved by blowing up $Y_{1} \times Y_{2} \subset X_{1} \times X_{2}$, where $Y_{1}=X_{1} \cap(y=0)$ and $Y_{2}=X_{2} \cap(z=0)$. By induction, $Y_{1}$ and $Y_{2}$ have finite-dimensional motive, and so $X$ (being dominated by something with finite-dimensional motive) has finite-dimensional motive.

Let $X \subset \mathbb{P}^{5 r+6}$ be a cubic as in (iii). Applying proposition 6 , we find a dominant rational map

$$
\phi: X_{1} \times X_{2} \rightarrow X,
$$

where

$$
\begin{aligned}
& X_{1}=\left\{f_{0}+f_{1}+\cdots+f_{r}+y^{3}=0\right\}, \\
& X_{2}=\left\{f_{r+1}\left(x_{5 r+5}, x_{5 r+6}\right)+z^{3}=0\right\} .
\end{aligned}
$$

The cubic $X_{1}$ has finite-dimensional motive because we have just proven (ii) for $r$, and $X_{2}$ has finitedimensional motive by the induction base. The indeterminacy of the rational map $\phi$ is resolved by blowing up $Y_{1} \times Y_{2} \subset X_{1} \times X_{2}$, where $Y_{1}=X_{1} \cap(y=0)$ and $Y_{2}=X_{2} \cap(z=0)$. The cubics $Y_{1}$ and $Y_{2}$ have finite-dimensional motive (by (i) for $r$ and the induction base), and so $X$ has finite-dimensional motive.

Next, let $X \subset \mathbb{P}^{5 r+2}$ be a cubic as in (iv). Applying proposition 6 , we find a dominant rational map

$$
\phi: X_{1} \times X_{2} \rightarrow X,
$$

where

$$
\begin{aligned}
& X_{1}=\left\{f_{0}+f_{1}+\cdots+f_{r-1}+y^{3}=0\right\}, \\
& X_{2}=\left\{f_{r}\left(x_{5 r}, x_{5 r+1}, x_{5 r+2}\right)+z^{3}=0\right\} .
\end{aligned}
$$

The cubics $X_{1}$ and $X_{2}$ have finite-dimensional motive by induction. The indeterminacy of the rational map $\phi$ is resolved by blowing up $Y_{1} \times Y_{2} \subset X_{1} \times X_{2}$, where $Y_{1}=X_{1} \cap(y=0)$ and $Y_{2}=X_{2} \cap(z=0)$. The cubics $Y_{1}$ and $Y_{2}$ have finite-dimensional motive (by induction), and so $X$ has finite-dimensional motive.

Finally, let $X \subset \mathbb{P}^{5 r+3}(\mathbb{C})$ be as in (v). There is a dominant rational map

$$
\phi: X_{1} \times X_{2} \rightarrow X,
$$

where

$$
\begin{aligned}
& X_{1}=\left\{f_{0}+f_{1}+\cdots+f_{r-1}+y^{3}=0\right\}, \\
& X_{2}=\left\{f_{r}\left(x_{5 r}, \ldots, x_{5 r+3}\right)+z^{3}=0\right\} .
\end{aligned}
$$

The indeterminacy of $\phi$ is resolved by blowing up $Y_{1} \times Y_{2} \subset X_{1} \times X_{2}$, where $Y_{1}=X_{1} \cap(y=0)$ and $Y_{2}=X_{2} \cap(z=0)$. The varieties $X_{1}, X_{2}, Y_{1}, Y_{2}$ all have finite-dimensional motive, and so $X$ has finite-dimensional motive. This closes the proof. 
Remark 7 In [19], using a similar argument I prove that certain quartic hypersurfaces have finite-dimensional motive.

We also mention, in passing, the following result concerning Chow groups of cubics: Let $X \subset \mathbb{P}^{n+1}(\mathbb{C})$ be a smooth cubic of type

$$
f_{0}\left(x_{0}, x_{1}, x_{2}\right)+f_{1}\left(x_{3}, x_{4}, x_{5}\right)+\cdots+f_{r}\left(x_{3 r}, x_{3 r+1}, x_{3 r+2}\right)+f_{r+1}\left(x_{3 r+3}, \ldots, x_{n+1}\right)=0,
$$

where $r=\left\lfloor\frac{n+1}{3}\right\rfloor$. Then Colliot-Thélène [5] has proven (exploiting the Shioda trick) that $X$ has universally trivial Chow group of 0 -cycles (i.e., there is an integral decomposition of the diagonal).

\section{The Fano variety of lines}

Corollary 8 Let $X \subset \mathbb{P}^{n+1}(\mathbb{C})$ be a cubic as in theorem $[5$ and let $F(X)$ denote the Fano variety of lines on $X$. Then $F(X)$ has finite-dimensional motive (of abelian type).

Proof This follows from [18].

Corollary 9 Let $X \subset \mathbb{P}^{n+1}(\mathbb{C})$ be a cubic as in theorem [5] and let $F(X)$ denote the Fano variety of lines on $X$. Then there exists a Chow-Künneth decomposition for $F(X)$, i.e. a set of mutually orthogonal idempotents in $A^{2 n-4}(F(X) \times F(X))$ summing to the diagonal and lifting the Künneth components.

Proof Thanks to the inclusion as a direct summand

$$
h(F(X)) \subset h\left(X^{[2]}\right)(-2) \text { in } \mathcal{M}_{\text {hom }}
$$

(where $X^{[2]}$ is the Hilbert scheme) [18], we know that $F(X)$ satisfies the Lefschetz standard conjecture (since $X^{[2]}$ does so). In particular, the Künneth components of the diagonal of $F(X)$ are algebraic [16]. Nilpotence then allows to lift the Künneth components to a Chow-Künneth decomposition [11, Lemma 3.1].

Remark 10 The existence of a Chow-Künneth decomposition for all smooth projective varieties is conjectured by Murre [20]. For any cubic fourfold $X$, Shen and Vial [27] have explicitly constructed a ChowKünneth decomposition for the Fano variety of lines $F(X)$. The argument is very different, since finitedimensionality is not known for the Fano variety of a general cubic fourfold.

\section{Voevodsky's conjecture}

Definition 11 (Voevodsky [33]) Let $X$ be a smooth projective variety. A cycle $a \in A^{r}(X)$ is called smash-nilpotent if there exists $m \in \mathbb{N}$ such that

$$
a^{m}:=\underbrace{a \times \cdots \times a}_{(m \text { times })}=0 \text { in } A^{m r}(X \times \cdots \times X) .
$$

Two cycles $a, a^{\prime}$ are called smash-equivalent if their difference $a-a^{\prime}$ is smash-nilpotent. We will write $A_{\otimes}^{r}(X) \subset A^{r}(X)$ for the subgroup of smash-nilpotent cycles.

Conjecture 12 (Voevodsky [33]) Let $X$ be a smooth projective variety. Then

$$
A_{\text {num }}^{r}(X) \subset A_{\otimes}^{r}(X) \text { for all } r .
$$

Remark 13 It is known [1. Théorème 3.33] that conjecture 12] implies (and is strictly stronger than) conjecture 2] For partial results concerning conjecture 12, cf. [25], [26], [29, Theorem 3.17].

As a corollary of finite-dimensionality, we can verify Voevodsky's conjecture for all odd-dimensional cubics as in theorem 5 
Corollary 14 Let $X \subset \mathbb{P}^{n+1}(\mathbb{C})$ be a smooth cubic as in theorem [5] Assume $n$ is odd. Then

$$
A_{\text {num }}^{r}(X) \subset A_{\otimes}^{r}(X) \text { for all } r .
$$

Proof As $X$ is a hypersurface, the Künneth components $\pi_{j}$ are algebraic [16] and the Chow motive of $X$ decomposes

$$
h(X)=h_{n}(X) \oplus \bigoplus_{j} \mathbb{L}\left(n_{j}\right) \text { in } \mathcal{M}_{\text {rat }} .
$$

(Here $\mathbb{L}$ denotes the Lefschetz motive, and the motive $h_{n}(X)$ is defined simply as $\left(X, \Delta-\sum_{j \neq n} \pi_{i}, 0\right)$.) Since $A_{\text {num }}^{r}\left(\mathbb{L}\left(n_{j}\right)\right)=0$, we have

$$
A_{\text {num }}^{r}(X)=A_{\text {num }}^{r}\left(h_{n}(X)\right) .
$$

The motive $h_{n}(X)$ is oddly finite-dimensional. (Indeed, since $n$ is odd we have that the motive $\operatorname{Sym}^{m} h_{n}(X) \in$ $\mathcal{M}_{\text {hom }}$ is 0 for some $m>>0$. By finite-dimensionality, the same then holds in $\mathcal{M}_{\text {rat }}$.) The proposition now follows from the following result (which is [15, Proposition 6.1], and which is also applied in [13] where I learned this):

Proposition 15 (Kimura [15]) Suppose $M \in \mathcal{M}_{\text {rat }}$ is oddly finite-dimensional. Then

$$
A^{r}(M) \subset A_{\otimes}^{r}(M) \text { for all } r .
$$

Acknowledgements This note is a belated echo of the Strasbourg 2014-2015 groupe de travail based on the monograph [35]. Thanks to all the participants for the pleasant and stimulating atmosphere. Many thanks to Yasuyo, Kai and Len for lots of enjoyable after-work apéritifs.

\section{References}

1. Y. André, Motifs de dimension finie (d'après S.-I. Kimura, P. O’Sullivan,...), Séminaire Bourbaki 2003/2004, Astérisque 299 Exp. No. 929, viii, $115-145$,

2. A. Beauville, Sur l'anneau de Chow d'une variété abélienne, Math. Ann. 273 (1986), 647—651,

3. M. Brion, Log homogeneous varieties, in: Actas del XVI Coloquio Latinoamericano de Algebra, Revista Matemática Iberoamericana, Madrid 2007, arXiv: math/0609669

4. M. de Cataldo and L. Migliorini, The Chow groups and the motive of the Hilbert scheme of points on a surface, Journal of Algebra 251 no. 2 (2002), 824-848,

5. J.-L. Colliot-Thélène, $\mathrm{CH}_{0}$-trivialité universelle d'hypersurfaces cubiques presque diagonales, Alg. Geometry,

6. P. Deligne, La conjecture de Weil pour les surfaces K3, Invent. Math. 15 (1972), 206-226,

7. V. Guletskil and C. Pedrini, The Chow motive of the Godeaux surface, in: Algebraic Geometry, a volume in memory of Paolo Francia (M.C. Beltrametti et alii, editors), Walter de Gruyter, Berlin New York, 2002,

8. F. Ivorra, Finite dimensional motives and applications (following S.-I. Kimura, P. O'Sullivan and others), in: Autour des motifs, Asian-French summer school on algebraic geometry and number theory, Volume III, Panoramas et synthèses, Société mathématique de France 2011,

9. J. Iyer, Murre's conjectures and explicit Chow-Künneth projectors for varieties with a nef tangent bundle, Transactions of the Amer. Math. Soc. 361 (2008), 1667-1681,

10. J. Iyer, Absolute Chow-Künneth decomposition for rational homogeneous bundles and for log homogeneous varieties, Michigan Math. Journal Vol.60, 1 (2011), 79—91,

11. U. Jannsen, Motivic sheaves and filtrations on Chow groups, in: Motives (U. Jannsen et alii, editors), Proceedings of Symposia in Pure Mathematics Vol. 55 (1994), Part 1,

12. U. Jannsen, On finite-dimensional motives and Murre's conjecture, in: Algebraic cycles and motives (J. Nagel and C. Peters, editors), Cambridge University Press, Cambridge 2007,

13. B. Kahn and R. Sebastian, Smash-nilpotent cycles on abelian 3-folds, Math. Res. Letters 16 (2009), 1007-1010,

14. T. Katsura and T. Shioda, On Fermat varieties, Tohoku Math. J. Vol. 31 No. 1 (1979), 97-115,

15. S. Kimura, Chow groups are finite dimensional, in some sense, Math. Ann. 331 (2005), 173-201,

16. S. Kleiman, The standard conjectures, in: Motives (U. Jannsen et alii, editors), Proceedings of Symposia in Pure Mathematics Vol. 55 (1994), Part 1,

17. R. Laterveer, A family of cubic fourfolds with finite-dimensional motive, to appear in Journal Math. Soc. Japan,

18. R. Laterveer, A remark on the motive of the Fano variety of lines of a cubic, Ann. Math. Québec 41 no. 1 (2017), 141-154,

19. R. Laterveer, Some elementary examples of quartics with finite-dimensional motive, Annali dell'Universita di Ferrara, doi:10.1007/s11565-016-0263-x,

20. J. Murre, On a conjectural filtration on the Chow groups of an algebraic variety, parts I and II, Indag. Math. 4 (1993), 177-201, 
21. J. Murre, J. Nagel and C. Peters, Lectures on the theory of pure motives, Amer. Math. Soc. University Lecture Series 61, Providence 2013,

22. C. Pedrini, On the finite dimensionality of a $K 3$ surface, Manuscripta Mathematica 138 (2012), 59—72,

23. C. Pedrini and C. Weibel, Some surfaces of general type for which Bloch's conjecture holds, to appear in: Period Domains, Algebraic Cycles, and Arithmetic, Cambridge Univ. Press, 2015,

24. T. Scholl, Classical motives, in: Motives (U. Jannsen et alii, editors), Proceedings of Symposia in Pure Mathematics Vol. 55 (1994), Part 1,

25. R. Sebastian, Smash nilpotent cycles on varieties dominated by products of curves, Comp. Math. 149 (2013), 1511-1518,

26. R. Sebastian, Examples of smash nilpotent cycles on rationally connected varieties, Journal of Algebra 438 (2015), 119129 ,

27. M. Shen and C. Vial, The Fourier transform for certain hyperKähler fourfolds, Memoirs of the AMS 240 (2016), no.1139,

28. T. Shioda, The Hodge conjecture for Fermat varieties, Math. Ann. 245 (1979), 175-184,

29. C. Vial, Projectors on the intermediate algebraic Jacobians, New York J. Math. 19 (2013), 793-822,

30. C. Vial, Remarks on motives of abelian type, to appear in Tohoku Math. J.,

31. C. Vial, Niveau and coniveau filtrations on cohomology groups and Chow groups, Proceedings of the LMS 106(2) (2013), $410-444$,

32. C. Vial, Chow-Künneth decomposition for 3- and 4-folds fibred by varieties with trivial Chow group of zero-cycles, J. Alg. Geom. 24 (2015), 51-80,

33. V. Voevodsky, A nilpotence theorem for cycles algebraically equivalent to zero, Internat. Math. Research Notices 4 (1995), $187-198$,

34. C. Voisin, Bloch's conjecture for Catanese and Barlow surfaces, J. Differential Geometry 97 (2014), 149—175,

35. C. Voisin, Chow Rings, Decomposition of the Diagonal, and the Topology of Families, Princeton University Press, Princeton and Oxford, 2014,

36. Z. Xu, Algebraic cycles on a generalized Kummer variety, arXiv:1506.04297 1 . 